\title{
L'Université des réseaux d'expression française au service des universités et des chercheurs francophones
}

\section{Michel Guillou}

* Voir à ce sujet, Francophonie Scientifique Le Tournant, collection Prospectives Francophones. Co-édition UREF/AUPELF/John Libbey Eurotext, 1989 : $184 \mathrm{p}$.

\section{ADRESSE}

M. Guillou : professeur, délégué général de l'Université des réseaux d'expression française (UREF). AUPELF/UREF, 192, boulevard

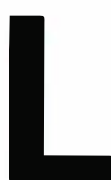

a construction d'un espace scientifique francophone est parvenue, ces deux dernières années, à un tournant*. Ou les Chefs d'État et de Gouvernement des pays ayant en commun l'usage du français l'engagent résolument avec des moyens importants et dans le cadre d'une stratégie globale, ou alors on a tout lieu de penser qu'elle ne se fera pas. En matière d'enseignement supérieur et de recherche, un pas essentiel a été fait avec la création de l'Université des réseaux d'expression française par les Sommets de Québec en 1987 et de Dakar en 1989. Programme majeur des Sommets, dont l'exécution a été confiée à l'Association des universités partiellement ou entièrement de langue française (AUPELF), l'UREF s'est vu confier le mandat de consolider l'espace scientifique francophone. Elle a pour ambition de nouer, dans tout le tissu scientifique de la francophonie, les liens de l'échange, de l'information et de la solidarité dans une perspective de développement et de modernité.

\section{Les enjeux sont clairs}

La recherche s'internationalise : elle se structure dans de grands ensembles régionaux ; obéit de plus en plus aux impératifs économiques et technologiques de grandes puissances, servies par des appareils contraignants d'évaluation des chercheurs. Pour entrer dans la compétition, se forger un avenir, la francophonie a l'obligation de constituer, entre tous les Etats qui partagent le français, une communauté économique, technologique et scientifique tout autant que culturelle et linguistique. Il lui faut ainsi se donner les outils nécessaires à cette ambition.

Nous avons pris, de longue date, des engagements envers l'Afrique. Nous avons soutenu que le français était, pour elle, l'outil de développement, le passage obligé pour l'accès à la science et à la technologie. Or, le fossé qui sépare les pays francophones les plus riches des plus déshérités s'accroît et menace la francophonie d'éclatement. Cette tendance doit s'inverser : il faut renforcer les infrastructures de la recherche, fixer en Afrique les chercheurs africains sur des grands pôles universitaires et de recherche, renforcer les centres et les équipes les plus performants, valoriser les résultats les plus avancés, donner ainsi à l'Afrique les moyens de la reconquête de sa créativité scientifique, dans le cadre d'un véritable partenariat avec les pays du Nord. L'Afrique a besoin d'un appui massif et volontariste. Continent associé au destin de l'Europe, elle est l'avenir de la francophonie.

Il faut également recréer les courants d'échange entre les communautés scientifiques francophones d'Europe et du Canada qui tendent ces dernières années à se tourner exclusivement vers des ensembles plus vastes, plus proches, que sont l'Europe et l'Amérique du Nord. 


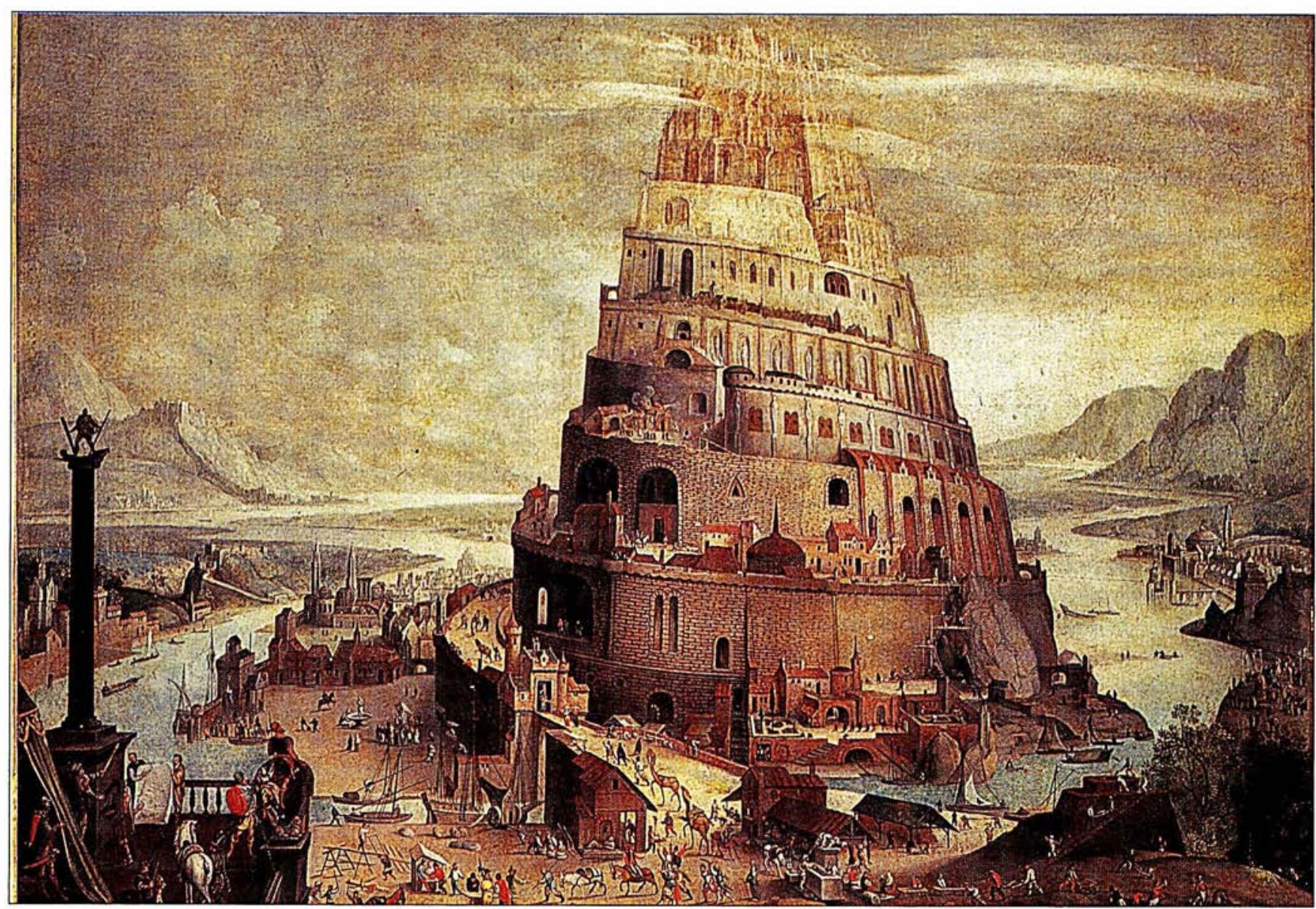

Tour de Babel. Jan Brueghel (1568-1625). Pour punir l'orgueil des hommes qui voulaient s'élever jusqu'à lui en construisant une grande tour, Dieu leur attribua des langages différents: les bâtisseurs cessèrent de se comprendre, et par conséquent ne purent poursuivre leur œuvre commune et insensée. (Brueghel, la Tour de Babel, Scala).

Parallèlement à l'usage de l'anglais comme lingua franca de la communication scientifique spécialisée, il faut donner au français toute la place qui est la sienne de grande langue de communication scientifique internationale. Pour ce faire, il convient de préserver sa capacité à garder toutes les fonctions d'une langue et à concourir à la création des codes scientifiques de demain.

Affermir le français comme langue d'innovation, ce n'est pas engager une lutte hégémonique dérisoire contre l'anglais: (1) c'est dans un authentique esprit de partenariat, garantir aux pays du Sud francophone la possibilité d'utiliser, avec la langue française, leur principale clef d'accès à la modernité ; (2) c'est préserver une certaine approche franco$\mathrm{m} / \mathrm{s} n^{\circ} 3$ vol. 6 , mars 90 phone de la science dans la mesure où la langue est le support de la pensée en même temps qu'un outil de communication, toute science conservant en effet l'empreinte de la culture qui la porte; (3) c'est enfin assurer, à terme, l'émergence des autres langues par le refus de l'uniformisation et l'affirmation du pluralisme.

A cet égard, la promotion du français dans les secteurs de l'innovation et de la créativité doit s'accompagner de la promotion, en Afrique, en Europe, en Amérique, du multilinguisme par l'usage égal des langues qui y sont principalement parlées. L'UREF propose, dans cet esprit, de fonder le Collège de l'Europe, qui serait un lieu de diffusion du savoir dans les grandes langues européennes de la science.
La réussite de tels engagements constitution d'un grand espace scientifique francophone, partenariat renforcé avec l'Afrique, utilisation du français à tous les niveaux de la communication scientifique, développement du multilinguisme - nécessite l'adhésion de la communauté scientifique internationale de langue française.

\section{La réponse}

Nous devons aux Chefs d'État et de Gouvernement réunis à Paris, à Québec et, plus récemment, à Dakar, d'avoir permis à la communauté francophone de prendre conscience de ces enjeux. Plus encore, ils ont doté cette francophonie moderni- 
sée et tournée vers l'avenir de nouveaux outils.

Opérateur multilatéral privilégié des Sommets pour l'enseignement supérieur et la recherche, mise en œuvre au sein de l'AUPELF, l'Université des réseaux d'expression française est l'un de ces outils.

C'est essentiellement à elle qu'il revient de consolider et d'animer l'espace scientifique francophone, de susciter une mobilisation des chercheurs et de créer un mouvement d'appartenance. A cette fin, elle doit mailler, mettre en réseaux l'espace scientifique et technique francophone, organiser à tous niveaux les échanges d'hommes, d'idées et de connaissances afin de désenclaver, de décloisonner la recherche et les chercheurs, favoriser l'excellence et la qualité de la recherche, préoccupations qui répondent tout à la fois aux préoccupations Nord-Sud, Sud-Sud et Nord-Nord.

L'UREF agit dans le cadre d'une politique contractuelle avec le Sommet qui lui a confié le soin d'engager, en 1990, vingt grands programmes qui s'articulent autour de trois axes mobilisateurs : l'information scientifique et technique, les réseaux d'échange et la formation partagée.

\section{Les moyens mis en cuvre}

L'information scientifique et technique (IST). L'IST circule mal entre le Nord et le Sud, notamment parce que les moyens mis en œuvre sont insuffisants ou inadaptés. Les programmes de l'UREF visent tous le même objectif : maintenir et développer l'édition et la diffusion la plus large possible de tous les supports d'IST en langue française, qu'ils soient écrits ou informatiques, par la collection Universités francophones de manuels universitaires, d'ouvrages de référence de $3^{\text {e }}$ cycle vendus à moitié prix dans les pays du Sud et largement diffusés dans toute la francophonie.

Une rapide relance de la production de revues de synthèse et de diffusion des résultats de la recherche est indispensable.

L'Université des réseaux y contribue en lançant deux périodiques. Sciences et changements planétaires: une revue multidisciplinaire, entièrement de langue française, consåcrée aux grands bouleversements de la planète et à leurs conséquences, dans tous les domaines environnementaux, sociaux, économiques et humains. Les Cahiers de la recherche francophone: une collection de revues thématiques de haut niveau avec pour objectif de valoriser la recherche qui se fait au Sud, de faire accéder les chercheurs francophones à la notoriété internationale. Les articles pourront être écrits dans toutes les langues utilisées dans la francophonie. Ils seront publiés en français avec de larges encadrés et un résumé en anglais. Chaque cahier comportera, par ailleurs, un volet encyclopédique de mises au point régulières relatives à la thématique scientifique du cahier.

Parallèlement à cette reconquête, il conviendra, dans les prochaines années, de consacrer les moyens nécessaires à l'élaboration, à côté des Current Contents américains, de nouveaux systèmes de référencement. Il faut, en particulier, permettre un référencement international des publications scientifiques de langue française et la valorisation, ainsi, dans l'évaluation des chercheurs, des travaux écrits en langue française.

Diffusion de livres, de revues, de banques de données. La diffusion est indissociable de l'édition. A côté du secteur marchand de livres et revues fournis à prix très réduit aux étudiants, enseignants et chercheurs africains, l'UREF met en place des programmes de remise gratuite d'ouvrages et revues aux étudiants ainsi qu'aux bibliothèques universitaires d'Afrique, du Maghreb, du Liban, d'Haïti, de l'océan Indien et bientôt de l'Asie du Sud-Est - Viatique pour la remise d'ouvrages professionnels aux étudiants des pays en développement entrant dans la vie professionnelle, 2500 nouveaux médecins, notamment ont bénéficié de ce programme. - Un livre par étudiant pour les étudiants en cours d'études. - Bibliothèque minimale pour l'attribution aux bibliothèques des institutions universitaires du Sud de revues et ouvrages récents.

Les réseaux de recherche. Une recherche ouverte sur le partage des connaissances et une vie universitaire institutionnelle dynamique sont essen- tielles à la consolidation d'un espace francophone de la science et de la technique.

C'est l'objectif que se fixent les réseaux thématiques de recherche et les programmes multidisciplinaires de l'Université.

Induisant un authentique partenariat universitaire sur des thèmes de recherche qui vont du SIDA et du paludisme aux littératures francophones en passant par le génie parasécheresse, la télédétection et l'entreprenariat, ces réseaux décloisonnent la recherche et rompent en particulier l'isolement des chercheurs du Sud. Fondés sur l'échange, ils sont le moyen privilégié d'un accès équitable et généralisé à l'information scientifique et technique.

La formation partagée. L'UREF, c'est aussi la formation. Les futures élites, les enseignants et les chercheurs doivent être formés au meilleur et avoir un accès égal au savoir donc aux moyens d'y parvenir, au Sud comme au Nord. C'est dans cet esprit que sont proposées des bourses d'excellence grâce auxquelles des chercheurs post-doctoraux, du Nord comme du Sud, peuvent enrichir leurs connaissances et leur formation dans des laboratoires d'autres pays francophones.

Par ailleurs, le programme CIME, à l'instar des programmes européens ERASMUS et COMETT, permet aux étudiants de $2^{\mathrm{e}}$ et $3^{\mathrm{e}}$ cycles de suivre des stages dans des entreprises d'autres pays francophones ou de poursuivre, dans d'autres universités de la francophonie, des études qui seront validées dans leur pays d'origine.

Pour renforcer les $3^{\mathrm{e}}$ cycles d'université, pour favoriser la diffusion dans l'espace francophone des savoirs et des savoir-faire au plus haut niveau, un programme de formation à distance par satellite, UNISAT (Université et Satellite) s'adresse aux universités et aux entreprises.

De même, l'Université des réseaux aide à la mise en place de filières francophones d'enseignements nécessaires au développement et de cursus co-diplômants associant des Universités du Nord et du Sud. Elle s'appuie, pour cela, sur les conférences francophones des responsables d'établissements. 
La francophonie scientifique s'ouvre naturellement aux chercheurs des pays situés en dehors de l'aire francophone, qui utilisent le français.

C'est pourquoi l'UREF participe à la formation des scientifiques de pays non francophones et les intègre à ses programmes comme correspondants ou chercheurs associés. Des actions sont conduites au bénéfice des pays anglophones de l'Afrique subsaharienne, ainsi que des pays d'Amérique Centrale et du Sud. Un effort spécifique vient d'être fait pour la Roumanie.

Pour une grande politique scientifique de la francophonie

Tels sont les grands axes d'une politique scientifique visant à consolider un espace scientifique indispensable au maintien d'une langue française porteuse d'avenir, de modernité, d'excellence. En dix ans, nous devons donner aux chercheurs francophones les raisons et les moyens d'utiliser le français dans leur travail ; conforter l'enseignement supérieur des pays du Sud en consolidant leurs systèmes nationaux de formation et de recherche; créer au Sud les centres d'excellence et les technopoles indispensables; redonner un souffle, une ambition et des moyens aux systèmes et aux réseaux documentaires francophones; développer un authentique partenariat entre les chercheurs.

A ce prix, un espace scientifique francophone moderne sera créé et donnera une puissante impulsion à l'enrichissement mutuel des pays partageant l'usage du français.

Le $5^{\mathrm{e}}$ anniversaire de médecine/sciences, qui a ouvert la voie, est une bonne occasion de rappeler les enjeux et les engagements

TIRÉS A PART

M. Guillou.

$\mathrm{m} / \mathrm{s} n^{\circ} 3$ vol. 6 , mars 90 